\title{
Axionphilic cosmological moduli
}

\author{
Kwang Sik Jeong $\odot^{1, *}$ and Wan Il Park $\oplus^{2, \dagger}$ \\ ${ }^{1}$ Department of Physics, Pusan National University, Busan 46241, Korea \\ ${ }^{2}$ Division of Science Education and Institute of Fusion Science, Jeon-buk National University, \\ Jeonju, Jeonbuk 54896, Korea
}

(Received 8 August 2021; accepted 25 October 2021; published 10 December 2021)

\begin{abstract}
We show that string moduli have axionphilic nature owing to the model-insensitive derivative interactions arising from the Kähler potential. The decay of a modulus into stringy axions occurs without suppression by the mass of final states. Interestingly, it turns out to hold in general not only for the scalar partner of the stringy axion but also for any other moduli. The decay into (pseudo)-Nambu-Goldstone bosons (NGBs) also avoids such mass suppression if the modulus is lighter than or similar in mass to the scalar partner of the NGB. Such axionphilic nature makes string moduli a natural source of an observable amount of dark radiation in string compactifications involving ultralight stringy axions, and possibly in extensions of the Standard Model that include a cosmologically stable NGB such as the QCD axion. In the latter case, the fermionic superpartner of the NGB can also contribute to the dark matter as a feebly interacting massive particle.
\end{abstract}

DOI: 10.1103/PhysRevD.104.123528

\section{INTRODUCTION}

String compactification involves many moduli with various masses. Their Planck-suppressed couplings make them long-lived, and typically the lightest one most relevant to cosmology. Displaced from the postinflationary potential minimum during inflation, generically, moduli are coherently generated after the end of inflation and come to dominate the energy density of the universe before their decay. The standard hot thermal universe for a successful big bang nucleosynthesis (BBN) then requires the lightest modulus to decay dominantly into the Standard Model (SM) particles well before the BBN epoch. Accordingly, the modulus has to be heavier than about $100 \mathrm{TeV}$ [1-3], and its non-SM decay channels should be suppressed at least by a couple of orders of magnitude relative to the SM ones.

As the minimal interaction of a modulus with matter fields, the coupling to matter kinetic terms is incapable of rendering the modulus to dominantly decay into the SM sector, questioning the compatibility of string moduli with a successful cosmology. This is because the modulus decay into matter particles is generally suppressed by the mass of final states, whereas the decay into gravitinos or stringy

\footnotetext{
ksjeong@pusan.ac.kr

†wipark@jbnu.ac.kr
}

Published by the American Physical Society under the terms of the Creative Commons Attribution 4.0 International license. Further distribution of this work must maintain attribution to the author(s) and the published article's title, journal citation, and DOI. Funded by SCOAP ${ }^{3}$. axions has no such suppression. The former may cause the moduli-induced gravitino problem [4-6], while the latter may lead to too much dark radiation [7-9]. A viable reheating after modulus domination thus necessitates specific interactions between the modulus and the SM sector. In the Kachru-Kallosh-Linde-Trivedi (KKLT) [10] and LARGE volume scenarios [11], which are the explicit realizations of four-dimensional de Sitter vacua with all string moduli stabilized, one may rely on modulus-dependent gauge couplings [12] or a modulus coupling to the Higgs bilinear operator in the Kähler potential [7-9].

In this paper we point out that the decay of a modulus into axions, which is induced by the derivative interactions arising from its coupling to the Kähler potential, can occur without suppression by the mass of final states. This is the case for the decay into stringy axions regardless of from which modulus the stringy axion comes. The decay into Nambu-Goldstone bosons (NGBs) also avoids mass suppression if the modulus is not much heavier than the scalar partner of the NGB, i.e., the saxion. All these features are insensitive to the details of moduli stabilization and string compactification, indicating that string moduli are basically axionphilic. Such axionphilic nature makes a crucial impact on cosmology because a sizable amount of dark radiation is produced from their decay in string compactifications giving ultralight stringy axions, and possibly in SM extensions including a cosmologically stable axion, like the QCD axion solving the strong $C P$ problem [13] or a light hidden photon.

Recovering the standard hot big bang universe for the successful BBN requires additional couplings to the SM 
sector if the universe undergoes a modulus-dominated phase. As mentioned, modulus-dependent gauge couplings and/or a modulus coupling to the holomorphic bilinear operator of SM-charged matter superfields in the Kähler potential may be necessary. On the other hand, if the modulus couples to a holomorphic bilinear operator involving the NGB superfield, NGBs are produced from the modulus decay at the rate without mass suppression. Also, the fermionic partner of the NGB can contribute to dark matter as a feebly interacting massive particle (FIMP). If sufficiently light, it may resolve the moduli-induced gravitino problem.

This paper is organized as follows. In Sec. II, we explore model-independent and -dependent interactions of a string modulus inducing its decay without mass suppression, and show that moduli are generally axionphilic insensitive to the details of model. The cosmological implications of the axionphilic nature of moduli are discussed in Sec. III. Section IV is the conclusions.

\section{MODULI INTERACTIONS}

String moduli generally couple to a (pseudo)-NGB and stringy axion, if it exists, through derivative interactions irrespective of the details of string compactification. We examine how significantly such interactions contribute to their decay. We also explore model-dependent derivative interactions, and discuss how to make moduli mainly decay into the SM sector as required for successful cosmology.

\section{A. Model-independent axionic decay modes}

It is well known that, for a string modulus stabilized while respecting the associated shift symmetry, the decay of its real component into a pair of its imaginary one, dubbed as stringy axion, is not mass suppressed [7-9]. It is also known that the decays of moduli into matter particles caused by derivative interactions arising from the Kähler potential are however typically suppressed by the mass of final states. In this work we show that, interestingly, such mass suppression does not occur for the decay into NGBs too if the scalar partner of the NGB, i.e., the saxion, is heavier than or similar in mass to the modulus. We further find that, regardless of from which modulus the stringy axion originates, moduli decay into stringy axions without mass suppression.

In order to see the effect of derivative interactions, we consider a simple model with a single modulus $S$ that enjoys a shift symmetry, $\operatorname{Im}(S) \rightarrow \operatorname{Im}(S)+$ constant, at the perturbative level. The shift symmetry is not essential for determining the modulus decay width, but makes our discussion simpler because it ensures that the modulus appears in the Kähler potential in the combination of $S+S^{*}$. The Kähler potential is written

$$
K=K_{0}+Z \Phi \Phi^{*},
$$

where $\Phi=\phi+\sqrt{2} \theta \psi+\theta \theta F$ represents a matter superfield, and we set the reduced Planck mass, $M_{\mathrm{Pl}}=1$, unless explicitly written. Here $K_{0}$ and $Z$ are a function of $S+S^{*}$. Expanding the modulus around its vacuum expectation value (VEV)

$$
\delta S \equiv S-S_{0}=s+\sqrt{2} \theta \tilde{s}+\theta \theta F^{S},
$$

one always finds the Kähler potential interaction of the modulus with matter superfields

$$
\left(\delta S+\delta S^{*}\right) \Phi \Phi^{*}
$$

regardless of the details of the model, where the overall coupling constant is proportional to $\left\langle\partial_{S} Z\right\rangle$. If matter fields do not develop a VEV, the modulus decay is determined simply by the interactions arising from the $D$ term of (3). In terms of the component fields, they read

$\left.\delta S \Phi \Phi^{*}\right|_{D}=s F F^{*}+\phi F^{S} F^{*}-s \phi \partial^{2} \phi^{*}-i s \psi \sigma^{\mu} \partial_{\mu} \bar{\psi}$,

omitting total derivatives. Written in the above form, the derivative interactions are directly combined with the equations of motion for $\phi$ and $\psi$ to show that the decay rate of the modulus is suppressed by the mass of decay products.

On the other hand, if $\phi$ develops a nonzero VEV, $\phi$ and $\psi$ are not the mass eigenstates anymore, and there also arises kinetic mixing between the moduli and matter fields. To see how the modulus decay is affected, we consider the case where $\phi$ spontaneously breaks a global U(1) symmetry. Then the matter scalar field is decomposed into the saxion and the NGB

$$
\phi(x)=\frac{f}{\sqrt{2}}\left(1+\frac{\sigma(x)}{f}\right) e^{i \frac{a(x)}{f}},
$$

with $f$ being the axion decay constant, while the modulus field is written

$$
s(x)=\frac{1}{\sqrt{2}}\left(s_{r}(x)+i s_{i}(x)\right),
$$

for the real scalars $s_{r}$ and $s_{i}$. The derivative interaction from $\delta S \Phi \Phi^{*}$,

$$
\Delta \mathcal{L}=\sqrt{2} \kappa\left(s \phi \partial^{2} \phi^{*}+\text { H.c. }\right),
$$

gives rise to the kinetic mixing terms and scalar cubic interactions

$$
\begin{gathered}
\left.\kappa^{-1} \Delta \mathcal{L}\right|_{2}=f s_{r} \partial^{2} \sigma+f s_{i} \partial^{2} a \\
\left.\kappa^{-1} \Delta \mathcal{L}\right|_{3}=-\frac{1}{2} a a \partial^{2} s_{r}+a \sigma \partial^{2} s_{i} \\
+\left(s_{r} \sigma-s_{i} a\right) \partial^{2} \sigma+\left(s_{r} a+s_{i} \sigma\right) \partial^{2} a,
\end{gathered}
$$


up to total derivatives. Here the overall coupling constant is determined by

$$
\kappa=-\frac{1}{\sqrt{2}}\left\langle\left(\partial_{S}^{2} K_{0}\right)^{-\frac{1}{2}} \partial_{S} \ln Z\right\rangle,
$$

and it is generally of the order unity. ${ }^{1}$ As will be shown shortly, the kinetic mixing has critical impacts on the modulus decay when combined with the cubic interactions arising from the matter Kähler potential,

$$
\left.\Phi \Phi^{*}\right|_{D, 3}=\frac{1}{2 f} a a \partial^{2} \sigma-\frac{1}{f} \sigma a \partial^{2} a,
$$

up to total derivatives.

The mixing terms (8) should be removed to examine how much the derivative interactions (9) contribute to the modulus decay. The kinetic mixing between the $\mathrm{CP}$-odd scalar bosons is eliminated by taking the field transformation,

$$
s_{i} \rightarrow s_{i}, \quad a \rightarrow a+\kappa f s_{i} .
$$

After the field transformation, i.e., in the canonical mass basis, all the derivative cubic couplings of $s_{i}$ vanish because the contributions from (9) and (11) exactly cancel each other. Hence there remain no interactions inducing the decay of $s_{i}$. For the $C P$-even scalar bosons, one can remove their kinetic mixing by taking the field transformation

$$
s_{r} \rightarrow s_{r}+\epsilon_{1} \kappa f \sigma, \quad \sigma \rightarrow \sigma+\epsilon_{2} \kappa f s_{r},
$$

for $f \ll M_{\mathrm{Pl}}$, with the coefficients determined by

$$
\epsilon_{1} \simeq-\frac{m_{\sigma}^{2}}{m_{s_{r}}^{2}-m_{\sigma}^{2}}, \quad \epsilon_{2} \simeq \frac{m_{s_{r}}^{2}}{m_{s_{r}}^{2}-m_{\sigma}^{2}},
$$

where $m_{i}$ denotes the mass of the indicated scalar boson, and we have neglected small corrections suppressed by $\left(f / M_{\mathrm{Pl}}\right)^{2}$. As a result, from (9) and (11), the derivative interactions for $s_{r}$ read

$$
\left.\Delta \mathcal{L}\right|_{3}=\kappa s_{r} \sigma \partial^{2} \sigma+\frac{\kappa}{2} \frac{m_{\sigma}^{2}}{m_{s_{r}}^{2}-m_{\sigma}^{2}} a a \partial^{2} s_{r}
$$

in the canonical mass basis. Therefore, if $m_{s_{r}} \gg m_{\sigma}$, the equations of motion lead to that both $s_{r} \rightarrow a a$ and $s_{r} \rightarrow \sigma \sigma$ are suppressed by the saxion mass. On the other hand, if

\footnotetext{
${ }^{1}$ For Kähler moduli, $\kappa$ is fixed by the location of the corresponding matter field in extra dimensions. It is then possible to suppress $\kappa$, but up to by a loop factor because it receives quantum corrections from nonperturbative effects, string loops, and higher-order $\alpha^{\prime}$ corrections.
}

$m_{s_{r}} \ll m_{\sigma}$, i.e., if the saxion is heavier than the modulus, the relevant interaction for the modulus decay becomes ${ }^{2}$

$$
\left.\Delta \mathcal{L}\right|_{3} \ni-\frac{\kappa}{2} a a \partial^{2} s_{r}
$$

The above shows that the modulus gets axionphilic, and decays into NGBs at the rate

$$
\Gamma\left(s_{r} \rightarrow a a\right)=\frac{\kappa^{2}}{32 \pi} \frac{m_{s_{r}}^{3}}{M_{\mathrm{Pl}}^{2}},
$$

where the masses of final states have been neglected. It should be noted that nonsuppression of the axionic decay mode $s_{r} \rightarrow a a$, which holds as long as the modulus is lighter than or similar in mass to the scalar partner of the NGB, is a model-independent generic feature of string moduli.

The nonderivative interactions involving $F$ and $F^{S}$ in (4) also mediate the decay, which is however suppressed by the mass of final states or further. This can be understood as follows. The matter $F$ component is expanded in powers of $\phi$ as

$$
F=\langle F\rangle+\left\langle\partial_{\phi} F\right\rangle \phi+\left\langle\partial_{\phi^{*}} F\right\rangle \phi^{*}+\cdots,
$$

while the scalar mass, $m_{\phi}$, is determined by

$$
m_{\phi}^{2}=\left\langle\frac{\partial^{2} V}{\partial \phi \partial \phi^{*}}\right\rangle=\left|\left\langle\partial_{\phi} F\right\rangle\right|^{2}+\left|\left\langle\partial_{\phi^{*}} F\right\rangle\right|^{2}+\cdots,
$$

where $V$ is the full scalar potential, and the ellipsis denotes possible extra contributions. Hence, the coefficients of the linear terms in (18) are bounded by

$$
\left|\left\langle\partial_{\phi} F\right\rangle\right|,\left|\left\langle\partial_{\phi^{*}} F\right\rangle\right| \lesssim m_{\phi}
$$

barring cancellation among various contributions to the scalar mass. Expanded in terms of $s$ and $s^{*}$, the modulus $F$ component exhibits the similar feature.

Let us move on to stringy axions that appear in string compactification such that a modulus, say $T$, is stabilized while preserving the associated shift symmetry. The interactions responsible for the modulus decay come from the Kähler potential:

$$
\left(\delta S+\delta S^{*}\right)\left(\delta T+\delta T^{*}\right)^{2},
$$

where $\delta S$ and $\delta T$ are moduli fluctuations around the vacuum. The $D$ term of the holomorphic cubic term,

\footnotetext{
${ }^{2}$ Equation (14) is valid for $f / M_{\mathrm{Pl}} \ll\left|m_{s_{r}}^{2}-m_{\sigma}^{2}\right| /\left(m_{s_{r}}^{2}+m_{\sigma}^{2}\right)$. Although it is unlikely, if the masses of the modulus and saxion are close to each other, the coupling of $a a \partial^{2} s_{r}$ induced by the kinetic mixing can be much larger than $\kappa$. Such enhancement can make the coupling of $s_{r}$ to NGBs suppressed not by $M_{\mathrm{Pl}}$ but by $f$ around the maximal mixing.
} 
$\delta S \delta T \delta T$, includes derivative interactions, which are however summed to be a total derivative. The relevant derivative interactions from the above Kähler potential term read

$$
\left.\Delta \mathcal{L}\right|_{3}=\sqrt{2} \hat{\kappa}\left(\frac{1}{2} t t \partial^{2} s^{*}+s t \partial^{2} t^{*}\right)+\text { H.c. }
$$

up to total derivatives. The coupling constant is determined by

$$
\hat{\kappa}=-\frac{1}{\sqrt{2}}\left\langle\left(\partial_{S}^{2} K_{0}\right)^{-1 / 2}\left(\partial_{T}^{2} K_{0}\right)^{-1} \partial_{S} \partial_{T}^{2} K_{0}\right\rangle,
$$

which is of the order unity in general. Here $t$ is the scalar component of $\delta T$ and is decomposed as

$$
t=\frac{1}{\sqrt{2}}(\tau+i \varphi)
$$

for real scalars $\tau$ and $\varphi$. The stringy axion, $\varphi$, remains massless until one adds nonperturbative effects breaking the shift symmetry explicitly. It is easy to see that the derivative interactions are written in terms of the real scalar fields as

$$
\begin{aligned}
\left.\hat{\kappa}^{-1} \Delta \mathcal{L}\right|_{3}= & \frac{1}{2} \tau \tau \partial^{2} s_{r}-\frac{1}{2} \varphi \varphi \partial^{2} s_{r}+\varphi \tau \partial^{2} s_{i} \\
& +\left(\tau s_{r}-\varphi s_{i}\right) \partial^{2} \tau+\left(\tau s_{i}+\varphi s_{r}\right) \partial^{2} \varphi .
\end{aligned}
$$

The first three interactions are expected to give a significant contribution to the decays of $s_{r}$ and $s_{i}$, while the decay via the others is mass suppressed.

To estimate correctly how fast the modulus decays via the derivative interactions, we need to remove kinetic mixing induced by the Kähler potential $\left(\delta S+\delta S^{*}\right)\left(\delta T+\delta T^{*}\right)$. Combined with the derivative interactions from $\left(\delta S+\delta S^{*}\right)^{3}$, $\left(\delta S+\delta S^{*}\right)^{2}\left(\delta T+\delta T^{*}\right)$, and $\left(\delta T+\delta T^{*}\right)^{3}$, the kinetic mixing modifies the couplings of the moduli interactions involving $\partial^{2} s_{r}$ and $\partial^{2} s_{i}$. However, their values in the canonical mass basis generally remain the same order of magnitude because the kinetic mixing is independent of $\hat{\kappa}$, differently from the NGB case where the interactions (8) and (9) are all originated from the same single Kähler potential term. The modulus decay into stringy axions is therefore given by

$$
\Gamma\left(s_{r} \rightarrow \varphi \varphi\right)=\frac{\kappa^{\prime 2}}{32 \pi} \frac{m_{s_{r}}^{3}}{M_{\mathrm{Pl}}^{2}},
$$

where $\kappa^{\prime}$, which depends on $\hat{\kappa}$ and kinetic mixing between the moduli, is generally of the order unity. Note that this nonsuppression of modulus decay into stringy axions is a quite general feature of string compactification, and holds regardless of which one of $s_{r}$ and $\tau$ is heavier.

The importance of modulus decay to stringy axions has been noticed for the case where $S$ and $T$ are identical, in the
LARGE volume scenario [7,8], and later in the generalized setup [9]. It is worth noting that, if string compactification involves a very light stringy axion, not only its scalar partner but also any other moduli decay into stringy axions with a sizable branching fraction insensitively to the details of moduli stabilzation. For instance, a massless stringy axion arises in the generalized KKLT with multiple Kähler moduli if a modulus, which does not appear in the superpotential, is stabilized by Kähler mixing with the others [14]. In such a case, the decay into stringy axions is sizable for all the real components of moduli.

\section{B. Model-dependent decay modes}

In this subsection we explore model-dependent interactions considerably contributing to the modulus decay, and discuss how to make the modulus mainly decay into the SM sector. Let us begin with a modulus coupling to the holomorphic bilinear operator of matter superfields,

$$
\left(\delta S+\delta S^{*}\right)\left(\Phi_{1} \Phi_{2}+\text { H.c. }\right),
$$

in the Kähler potential. It is the derivative interactions including only the scalar fields that can enhance the modulus decay. Whereas the holomorphic cubic term, $\delta S \Phi_{1} \Phi_{2}$, is irrelevant because its $D$ term only gives a total derivative, the nonholomorphic cubic term gives

$$
\left.\delta S^{*} \Phi_{1} \Phi_{2}\right|_{D}=-\phi_{1} \phi_{2} \partial^{2} s^{*},
$$

up to total derivatives. It is obvious that the decay of the modulus via the interaction (28) is not suppressed by the mass of final states. Hence, an interaction of the type (27) can enhance the modulus decay into the SM sector if the involved matter fields are SM charged.

More concretely, one can consider a modulus coupling to $Q Q^{c}$ in the Kähler potential with a coupling constant of the order unity or larger. Here the matter superfields $Q+Q^{c}$ are vectorlike under the SM gauge groups, and are lighter than the modulus. A natural candidate of such operators is the Higgs bilinear $H_{u} H_{d}$ [7-9], where $H_{u}$ and $H_{d}$ are, respectively, the up- and down-type Higgs doublet. The modulus coupling to $H_{u} H_{d}$ in the Kähler potential

$$
\Delta K_{1}=\xi H_{u} H_{d}+\text { H.c. }
$$

includes the term

$$
\left\langle\partial_{S} \xi\right\rangle\left(\delta S+\delta S^{*}\right) H_{u} H_{d}+\text { H.c., }
$$

where $\xi$ is a function of $S+S^{*}$. Meanwhile, it gives a contribution to the Higgsino mass parameter $\mu$ and the Higgs mixing parameter $B$ as

$$
\Delta \mu=\langle\xi\rangle m_{3 / 2}, \quad \Delta B=-m_{3 / 2},
$$


with $m_{3 / 2}$ being the gravitino mass, because it explicitly breaks the super-Weyl symmetry. It is then difficult to avoid the Higgs $\mu / B \mu$ problem in the scenario where the gravitino is much heavier than the visible sparticles, i.e., if anomaly mediation, which is a model-independent source of soft supersymmetry-breaking masses in supergravity, is sizable as is the case in the KKLT moduli stabilization. To allow an order unity coupling of $\delta S$ to $H_{u} H_{d}$ while suppressing the contributions to $\mu$ and $B$, one can consider

$$
\Delta K_{2}=\xi \frac{X^{*}}{X} H_{u} H_{d}+\text { H.c. },
$$

for a singlet $X$ that is radiatively stabilized by the loop potential [12]. As induced by the above super-Weyl invariant Kähler potential term, both $\Delta \mu$ and $\Delta B$ are loop suppressed relative to $m_{3 / 2}$ as required for the correct electroweak symmetry breaking, while the modulus coupling to $H_{u} H_{d}$ is still given by $\left\langle\partial_{S} \xi\right\rangle$ and is generally of the order unity.

Let us examine how the modulus decay is affected by the interaction (27) when both $\phi_{1}$ and $\phi_{2}$ develop a VEV to spontaneously break the global U(1) symmetry, under which $\phi_{1}$ and $\phi_{2}$ carry charge 1 and -1 , respectively, so that $\phi_{1} \phi_{2}$ becomes U(1) invariant. The scalar fields are decomposed as

$$
\phi_{i}=\frac{f_{i}}{\sqrt{2}}\left(1+\frac{\sigma_{i}}{f_{i}}\right) e^{i \frac{a_{i}}{f_{i}}}
$$

for $i=1,2$. The massless NGB is then written

$$
a=\frac{f_{1} a_{1}-f_{2} a_{2}}{\sqrt{f_{1}^{2}+f_{2}^{2}}} .
$$

Through the interaction (28), the modulus couples to the two massive scalar bosons composed of $\sigma_{i}$ and the other combination of $a_{i}$ proportional to $f_{2} a_{1}+f_{1} a_{2}$, but it is forbidden to couple to the NGB due to the U(1) symmetry. Nonetheless, in the presence of the coupling (28), NGBs can be produced abundantly from the cascade decay of moduli because the saxion generally decays mainly into NGBs via the $a a \partial^{2} \sigma$ interaction.

Another role of the interaction (28) is to induce kinetic mixing between the modulus and $\sigma_{i}$ after spontaneous $\mathrm{U}(1)$ breaking. Combined with the interactions $a_{i} a_{i} \partial^{2} \sigma_{i}$ coming from the kinetic term of $\phi_{i}$, such kinetic mixing generates the $a a \partial^{2} s_{r}$ interaction whose coupling is given by

$$
\frac{2 f_{1} f_{2}}{f_{1}^{2}+f_{2}^{2}}
$$

times the coupling of (28), for the case where the modulus is much heavier than the massive scalar bosons composed of $\sigma_{i}$. This implies that, after electroweak symmetry breaking, the modulus coupling to $H_{u} H_{d}$ in the Kähler potential induces the modulus decay into the $C P$ even and odd neutral Higgs bosons, and the charged Higgs bosons without mass suppression. In addition, due to the kinetic mixing, it contributes to the modulus decay into the associated NGBs, i.e., into the longitudinal modes of the weak gauge bosons, according to the Goldstone boson equivalence theorem $[15,16]$.

As a model-dependent modulus decay mode, one can also consider the decay into the gauge sector, which is possible if the modulus appears in the gauge kinetic function:

$$
f_{a}=S+\Delta f_{a},
$$

with $\Delta f_{a}$ being an $S$-independent constant. It is straightforward to see that the decay rate into gauge bosons reads [12]

$$
\Gamma\left(s_{r} \rightarrow g g\right)=\frac{N_{g}}{128 \pi} \kappa_{g}^{2} \frac{m_{s_{r}}^{3}}{M_{\mathrm{Pl}}^{2}},
$$

where $N_{g}=12$ counts the number of gauge bosons, and the order unity constant $\kappa_{g}$ is determined by

$$
\kappa_{g}=\left\langle\left(\partial_{S}^{2} K_{0}\right)^{-\frac{1}{2}} \frac{1}{\operatorname{Re} f_{a}}\right\rangle .
$$

On the other hand, the decay rate into gauginos is written

$$
\Gamma\left(s_{r} \rightarrow \tilde{g} \tilde{g}\right)=\beta_{r}^{2} \Gamma\left(s_{r} \rightarrow g g\right),
$$

with $\beta_{r}$ defined by

$$
\left\langle\partial_{s} F^{S}\right\rangle s+\left\langle\partial_{s^{*}} F^{S}\right\rangle s^{*} \equiv \beta_{r} m_{s_{r}} s_{r}+\beta_{i} m_{s_{i}} s_{i},
$$

where $\beta_{r}$ and $\beta_{i}$ do not exceed order unity in size. Here the decay rates have been evaluated neglecting the mass of decay products. The decay rates of $s_{i}$ can be read off from those for $s_{r}$ by taking the replacements, $m_{s_{r}} \rightarrow m_{s_{i}}$ and $\beta_{r} \rightarrow \beta_{i}$. Note that the KKLT leads to $\beta_{r} \simeq \beta_{i} \simeq 1$ for $\Delta f_{a}=0$. In order to enhance the decay into the visible sector further, one may consider the case where the Higgsino mass parameter is generated from the Kähler potential as $(29)[7,8]$ or as $(32)$.

We close this section by mentioning that the modulus can decay to a gravitino pair at a sizable rate if kinematically allowed. In particular, the decay is dominated by the coupling to the helicity $\pm 1 / 2$ components, i.e., to the Goldstino, which is proportional to the modulus $F$ term [4-6]. The decay rate into gravitinos reads

$$
\Gamma\left(s_{r} \rightarrow \tilde{G} \tilde{G}\right)=\frac{\kappa_{3 / 2}^{2}}{288 \pi} \frac{m_{s_{r}}^{3}}{M_{\mathrm{Pl}}^{2}},
$$


in the limit $m_{3 / 2} \ll m_{s_{r}}$, with $m_{3 / 2}$ being the gravitino mass. Here $\kappa_{3 / 2}$ is defined as follows:

$$
\kappa_{3 / 2} \equiv\left\langle\left(\partial_{S}^{2} K_{0}\right)^{\frac{1}{2}} F^{S}\right\rangle \frac{m_{s_{r}}}{m_{3 / 2}^{2}},
$$

whose size is below order unity because the modulus interactions are gravitational. If it is of the order unity, as is typically the case in the known examples of moduli stabilization, a large gravitino yield after moduli decay can cause cosmological difficulties, dubbed as the moduliinduced gravitino problem.

\section{COSMOLOGICAL IMPLICATIONS}

Reheating takes place via moduli decay if the universe passes through a modulus-dominated epoch as generally expected in string compactifications. To catch the relevant features of such driven reheating, we simply consider a single modulus $S$ under the assumption that its real and imaginary component have a similar mass, $m_{s} .{ }^{3}$ In flux compactifications, a modulus unfixed by flux can be stabilized by nonperturbative dynamics or Kähler mixing with others. Its mass is then tied to the scale of supersymmetry breaking, $m_{s} \sim m_{3 / 2}$, up to a factor of order $\ln \left(M_{\mathrm{Pl}} / m_{3 / 2}\right)$ [17,18]. On the other hand, in the LARGE volume scenario, the mass of the large volume modulus can be much smaller than $m_{3 / 2}$ [11].

To allow successful BBN after the modulus-dominated phase, the modulus should decay dominantly into the visible sector while forming the standard thermal background with temperature $T_{s}$ constrained by $[19,20]$

$$
T_{s} \simeq\left(\frac{90}{\pi^{2} g_{*}}\right)^{\frac{1}{4}}\left(\Gamma_{s} M_{\mathrm{Pl}}\right)^{\frac{1}{2}} \gtrsim 5 \mathrm{MeV},
$$

where $g_{*}$ is the relativistic degrees of freedom at $T_{s}$, and $\Gamma_{s}$ is the total decay width of the modulus. Here we have assumed an instantaneous conversion of the modulus energy density to radiation. The constraint on $T_{s}$ requires the modulus to be heavier than about $100 \mathrm{TeV}$. Modulus domination also constrains models for baryon and dark matter genesis because a huge amount of entropy is released from moduli decay. If the genesis occurs at the early stage of moduli domination or before, the dilution

\footnotetext{
${ }^{3}$ For the case of multiple moduli, the decay properties discussed in Sec. II apply to all the moduli. If not much heavier than the lightest one, heavy moduli can still be cosmologically important. For instance, if there were two moduli, $S_{1}$ and $S_{2}$, with masses $m_{1}$ and $m_{2}\left(m_{1}<m_{2}\right)$, respectively, the energy density of the decay products of $S_{2}$ is diluted by the entropy released from late-time decay of $S_{1}$. The dilution factor is given by the ratio between their decay temperatures, and reads $\Delta \sim\left(m_{2} / m_{1}\right)^{3 / 2}$, assuming that the displacement of moduli at the onset of coherent oscillation is of the order of the Planck scale.
}

factor naively reads $\Delta \sim 10^{12}\left(m_{s} / \mathrm{PeV}\right)^{-1}$, since the initial displacement of the modulus after inflation is generally of the order of the Planck scale.

A viable reheating requires the modulus to decay dominantly to the visible sector. This can be achieved by adding model-dependent modulus couplings such as a coupling to the bilinear operator $Q Q^{c}$ or $H_{u} H_{d}$ in the Kähler potential, or to the visible gauge sector as discussed in Sec. II B. In the presence of such couplings, the decay rate into the visible sector reads

$$
\Gamma_{s \rightarrow \mathrm{SM}}=\frac{\kappa_{\mathrm{SM}}^{2}}{32 \pi} \frac{m_{s}^{3}}{M_{\mathrm{Pl}}^{2}},
$$

where $\kappa_{\mathrm{SM}}$ is a constant of order unity. As discussed already, the modulus generally decays into axions without mass suppression if string compactification involves a light stringy axion, $\varphi$, or if there is a (pseudo)-NGB, $a$, whose scalar partner has a mass larger than or comparable to the modulus mass. A theoretically well-motivated NGB is the QCD axion [21]. The branching ratio of the axionphilic modulus into axions is written

$$
\mathrm{Br}(s \rightarrow \text { axions })=\frac{\kappa^{2}+\kappa^{\prime 2}}{\kappa_{\mathrm{SM}}^{2}},
$$

which naturally lies in the range of the order of 0.1 . If the axions are cosmologically stable, the produced axions form dark radiation. The contribution to the effective number of neutrino species is estimated by [22]

$$
\Delta N_{\text {eff }}=\frac{43}{7} \frac{\operatorname{Br}(s \rightarrow \text { axions })}{1-\operatorname{Br}(s \rightarrow \text { axions })}\left(\frac{g_{*}\left(T_{\nu, \mathrm{dec}}\right)}{g_{*}\left(T_{s}\right)}\right)^{\frac{1}{3}},
$$

under the assumption that the branching ratio into the visible sector is given by $1-\operatorname{Br}(s \rightarrow$ axions $)$. Here $T_{\nu, \mathrm{dec}}$ is the neutrino decoupling temperature. An observable amount of dark radiation is a natural prediction of the axionphilic modulus insensitively to the detail of moduli stabilization. Dark radiation can have interesting cosmological effects in the early universe. For instance, if $\Delta N_{\text {eff }} \gtrsim 0.5$, which is the case for $\operatorname{Br}(s \rightarrow$ axions) larger than about 0.07 , it may relieve the Hubble tension $[23,24]$.

It should be noted that the presence of a plenitude of light stringy axions, an axiverse, has been suggested as evidence for the extra dimensions of string theory [25]. In the axiverse, the lightest modulus would dominantly decay into these axions, producing too much dark radiation if the universe undergoes a modulus-dominated phase. The modulus coupling to $H_{u} H_{d}$ or to the gauge sector would not be sufficient to suppress $\operatorname{Br}(s \rightarrow$ axions $)$. To avoid the moduli-induced axion problem, i.e., to enhance the modulus decay into the visible sector, one may thus rely on the modulus coupling to $Q Q^{c}$ in the Kähler potential, for instance, for a number of $Q+Q^{c}$ that form complete $\mathrm{SU}(5)$ multiplets. Here the supersymmetric mass of $Q+Q^{c}$ in the 
superpotential is assumed to be smaller than the modulus mass. We also note that, depending on their masses, the number of $Q+Q^{c}$ is constrained by the perturbativity of the gauge interactions up to the grand unification scale. The phenomenological and cosmological details of such particles are out of the scope of this paper, and hence will not be touched here.

The cosmological features discussed so far remain the same even if the saxion has an initial amplitude of the order of the Planck scale, as long as it decays much faster than the modulus, i.e., if

$$
m_{\sigma} \gtrsim 100 \mathrm{GeV}\left(\frac{f}{10^{12} \mathrm{GeV}}\right)^{\frac{2}{3}}\left(\frac{m_{s}}{\mathrm{PeV}}\right) .
$$

However, the situation changes much if the saxion is thermally trapped at the origin to drive thermal inflation [26]. It is also a plausible scenario although we do not consider it in this paper.

Moduli stabilization with $m_{s}>2 m_{3 / 2}$ generally confronts the moduli-induced gravitino problem unless one adds $R$-parity violating interactions. Interestingly, the gravitino problem can be relaxed if the fermionic partner of the axion, the axino, is much lighter than the lightest observable sparticle (LOSP) since otherwise LOSPs produced from gravitino decay would overclose the universe under the $R$-parity conservation. Even for moduli stabilization with $m_{s}<2 m_{3 / 2}$, the axino is still an attractive particle because, as a FIMP, it is a viable alternative to weakly interacting massive particle (WIMP) cold dark matter.

In the moduli-dominated universe, axinos are nonthermally produced from the decays of the modulus, gravitino, and LOSP. The axino abundance from LOSPs decay is quite model dependent because LOSPs can efficiently annihilate before their decay depending on the LOSP nature and the value of $f$. Meanwhile, independently of model details, the axino yield directly produced from the gravitationally interacting moduli and gravitinos is estimated by

$$
\left.Y_{\tilde{a}}\right|_{s \rightarrow \tilde{a}}=2 B_{\tilde{a}}^{s} \frac{3}{4} \frac{T_{s}}{m_{s}},
$$

where $B_{\tilde{a}}^{s}$ is defined by

$$
B_{\tilde{a}}^{s} \equiv \operatorname{Br}(s \rightarrow \tilde{a} \tilde{a})+\operatorname{Br}(s \rightarrow \tilde{G} \tilde{G}) \operatorname{Br}(\tilde{G} \rightarrow \tilde{a}),
$$

with $\operatorname{Br}(s \rightarrow \tilde{a} \tilde{a}) \sim\left(m_{\tilde{a}} / m_{s}\right)^{2}$ as follows from (4). Thus, in the case with $m_{s}<2 m_{3 / 2}$, the axino should have mass,

$$
m_{\tilde{a}} \lesssim 1 \mathrm{TeV}\left(\frac{0.1 \mathrm{GeV}}{T_{s}}\right)^{\frac{1}{3}}\left(\frac{m_{s}}{\mathrm{PeV}}\right)
$$

not to exceed the observed dark matter density. On the other hand, if $m_{s}>2 m_{3 / 2}$, the gravitino produced from moduli uniformly decays to the axino and all lighter visible sparticles, and should be heavier than a few tens of $\mathrm{TeV}$ in order to decay before BBN [27]. The branching fraction of the modulus decay to gravitinos is generally sizable, indicating that the axino should be much lighter than the above bound. The axino mass and the LOSP nature may further be constrained by the axino free-streaming scale at the time of matter-radiation equality, which should be less than the order of $\mathrm{Mpc}$ to be consistent with the observations of Lyman- $\alpha$ forest [28]. Axinos are mostly produced from the decay of LOSPs as long as the number of the visible sparticles lighter than the gravitino is much larger than unity. Then, the free-streaming constraint is relaxed if the LOSP scattering rate is larger than its decay rate so that LOSPs become nonrelativistic before their decay. See Refs. [12,29] for some examples in the KKLT scenario. Finally, we note that axinos are also produced via freeze-in processes, which can be effective depending on the value of $f$ if $T_{s}$ is not much lower than the freeze-out temperature of the LOSP [29].

\section{CONCLUSIONS}

In this work we have showed that string moduli have axionphilic nature. Induced by the model-insensitive derivative interactions, the decay of a modulus into stringy axions occurs without suppression by the mass of decay products. Interestingly, we found that this feature holds not only for the scalar partner of the stringy axion but also for any other moduli. The decay into (pseudo)-NGBs also avoids mass suppression if the modulus is not much heavier than the saxion. The axionphilic nature makes string moduli a natural source of an observable amount of dark radiation in the universe that passes through a modulus-dominated era as is generally expected in scenarios of string compactification. Proper reheating would then require moduli to have additional couplings to enhance their decay into the SM sector so that a hot thermal bath composed of mostly SM particles is formed. For instance, the modulus can couple to the gauge sector if it appears in the gauge kinetic function. Another way is to add a modulus coupling to the holomorphic bilinear operator of matter superfields in the Kähler potential. It is also interesting to note that the fermionic superpartner of a NGB as well as coherently oscillating NGBs can be a viable alternative to WIMP dark matter.

\section{ACKNOWLEDGMENTS}

The authors thank Fuminobu Takahashi for helpful discussion. This work was supported by the National Research Foundation of Korea (NRF) grant funded by the Korea government: Grants No. 2018R1C1B6006061, No. 2021R1A4A5031460 (K. S. J.), No. 2017R1D1A1B06035959 (W. I. P.). 
[1] G. D. Coughlan, W. Fischler, E. W. Kolb, S. Raby, and G. G. Ross, Phys. Lett. 131B, 59 (1983).

[2] J. R. Ellis, D. V. Nanopoulos, and M. Quiros, Phys. Lett. B 174, 176 (1986).

[3] A. S. Goncharov, A. D. Linde, and M. I. Vysotsky, Phys. Lett. 147B, 279 (1984).

[4] M. Endo, K. Hamaguchi, and F. Takahashi, Phys. Rev. Lett. 96, 211301 (2006).

[5] S. Nakamura and M. Yamaguchi, Phys. Lett. B 638, 389 (2006).

[6] M. Dine, R. Kitano, A. Morisse, and Y. Shirman, Phys. Rev. D 73, 123518 (2006).

[7] M. Cicoli, J. P. Conlon, and F. Quevedo, Phys. Rev. D 87, 043520 (2013).

[8] T. Higaki and F. Takahashi, J. High Energy Phys. 11 (2012) 125.

[9] T. Higaki, K. Nakayama, and F. Takahashi, J. High Energy Phys. 07 (2013) 005.

[10] S. Kachru, R. Kallosh, A. D. Linde, and S. P. Trivedi, Phys. Rev. D 68, 046005 (2003).

[11] V. Balasubramanian, P. Berglund, J. P. Conlon, and F. Quevedo, J. High Energy Phys. 03 (2005) 007.

[12] S. Nakamura, K. i. Okumura, and M. Yamaguchi, Phys. Rev. D 77, 115027 (2008).

[13] R. D. Peccei and H. R. Quinn, Phys. Rev. Lett. 38, 1440 (1977); Phys. Rev. D 16, 1791 (1977).

[14] K. Choi and K. S. Jeong, J. High Energy Phys. 01 (2007) 103.

[15] J. M. Cornwall, D. N. Levin, and G. Tiktopoulos, Phys. Rev. D 10, 1145 (1974); 11, 972(E) (1975).
[16] C. E. Vayonakis, Lett. Nuovo Cimento 17, 383 (1976).

[17] K. Choi, A. Falkowski, H. P. Nilles, and M. Olechowski, Nucl. Phys. B718, 113 (2005).

[18] K. Choi, K. S. Jeong, and K. I. Okumura, J. High Energy Phys. 07 (2008) 047.

[19] P. F. de Salas, M. Lattanzi, G. Mangano, G. Miele, S. Pastor, and O. Pisanti, Phys. Rev. D 92, 123534 (2015).

[20] T. Hasegawa, N. Hiroshima, K. Kohri, R. S. L. Hansen, T. Tram, and S. Hannestad, J. Cosmol. Astropart. Phys. 12 (2019) 012.

[21] For recent reviews, see, for instance, A. Ringwald, Phys. Dark Universe 1, 116 (2012); M. Kawasaki and K. Nakayama, Annu. Rev. Nucl. Part. Sci. 63, 69 (2013).

[22] K. Choi, E. J. Chun, and J. E. Kim, Phys. Lett. B 403, 209 (1997).

[23] A. G. Riess, S. Casertano, W. Yuan, L. M. Macri, and D. Scolnic, Astrophys. J. 876, 85 (2019).

[24] E. Di Valentino, O. Mena, S. Pan, L. Visinelli, W. Yang, A. Melchiorri, D. F. Mota, A. G. Riess, and J. Silk, Classical Quantum Gravity 38, 153001 (2021).

[25] A. Arvanitaki, S. Dimopoulos, S. Dubovsky, N. Kaloper, and J. March-Russell, Phys. Rev. D 81, 123530 (2010).

[26] D. H. Lyth and E. D. Stewart, Phys. Rev. Lett. 75, 201 (1995); Phys. Rev. D 53, 1784 (1996).

[27] M. Kawasaki, K. Kohri, T. Moroi, and Y. Takaesu, Phys. Rev. D 97, 023502 (2018).

[28] M. Viel, J. Lesgourgues, M. G. Haehnelt, S. Matarrese, and A. Riotto, Phys. Rev. D 71, 063534 (2005).

[29] K. J. Bae and K. S. Jeong, Phys. Rev. D 104, 015013 (2021). 